\title{
REVISED Use of cidofovir in pediatric patients with adenovirus
}

\section{infection [version 2; peer review: 3 approved]}

\author{
Lakshmi Ganapathi1,2, Alana Arnold³, Sarah Jones³, Al Patterson³, \\ Dionne Graham4 ${ }^{4}$ Marvin Harper ${ }^{1,2}$, Ofer Levy ${ }^{1,2}$
}

\author{
${ }^{1}$ Division of Infectious Diseases, Boston Children's Hospital, Boston, MA, USA \\ ${ }^{2}$ Harvard Medical School, Boston, MA, USA \\ ${ }^{3}$ Division of Pharmacy, Boston Children's Hospital, Boston, MA, USA \\ ${ }^{4}$ Program for Patient Quality and Safety, Boston Children's Hospital, Boston, MA, USA
}

\author{
V2 First published: 26 Apr 2016, 5:758 \\ https://doi.org/10.12688/f1000research.8374.1 \\ Latest published: 16 Dec 2016, 5:758 \\ https://doi.org/10.12688/f1000research.8374.2
}

\section{Abstract}

Background: Adenoviruses contribute to morbidity and mortality among immunocompromised pediatric patients including stem cell and solid organ transplant recipients. Cidofovir (CDV), an antiviral compound approved by the FDA in 1996, is used for treatment of adenoviral (ADV) infections in immunocompromised patients despite concern of potential nephrotoxicity.

Methods: We conducted a retrospective 5-year review at Boston Children's Hospital of 16 patients (mean age $=6.5$ years) receiving 19 courses of CDV. During therapy all pertinent data elements were reviewed to characterize potential response to therapy and incidence of renal dysfunction.

Results: Of the 19 CDV courses prescribed, 16 courses (84\%) were in patients who had a positive blood ADV Polymerase chain reaction (PCR) alone or in combination with positive ADV PCR/ Direct Immunofluorescence Assay (DFA) at another site. Respiratory symptoms with or without pneumonia were the most common presentation $(10 / 19,53 \%)$. In the majority of blood positive courses $(10 / 16,63 \%)$, viral clearance was also accompanied by clinical response. This was not the case in four courses where patients expired despite viral clearance, including one in which death was directly attributable to adenovirus. There was reversible renal dysfunction observed during the use of CDV.

Conclusions: CDV appeared safe and reasonably tolerated for treatment of ADV in this pediatric population and was associated with viral response and clinical improvement in the majority of patients but reversible renal dysfunction was a side effect. Further studies of the efficacy of CDV for immunocompromised children with ADV infection are warranted.
Open Peer Review

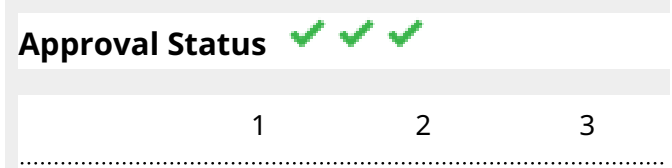

version 2

(revision)

16 Dec 2016

version 1

26 Apr 2016

1. Miguel O'Ryan (ID), Universidad de Chile,

Santiago, Chile

2. Jeffrey Bergelson, University of Pennsylvania School of Medicine, Philadelphia, USA

3. David A Hunstad, Washington University in

St Louis, St Louis, USA

Andrew Janowski, Washington University in

St Louis, St Louis, USA

Any reports and responses or comments on the article can be found at the end of the article. 


\section{Keywords}

cidofovir , anti-viral , pediatric, adenovirus, stem-cell, solid organ , immunocompromised

\section{Corresponding author: Ofer Levy (ofer.levy@childrens.harvard.edu)}

Competing interests: No competing interests were disclosed.

Grant information: OL's laboratory is supported by a Boston Children's Hospital Department of Medicine award to the Precision Vaccines Program as well as Global Health (OPPGH5284) and Grand Challenges Explorations (OPP1035192) awards from the Bill \& Melinda Gates Foundation and by NIH grants 1R01AI100135-01 and 3R01AI067353-05S1 and National Institute of Allergy \& Infectious Diseases Adjuvant Discovery Program, Contract No. HHSN272201400052C. The Levy Laboratory has received sponsored research support from VentiRx Pharmaceuticals, 3M Drug Delivery Systems, MedImmune, and Crucell (Johnson \& Johnson)- companies that develop adjuvants and vaccines.

The funders had no role in study design, data collection and analysis, decision to publish, or preparation of the manuscript.

Copyright: @ 2016 Ganapathi L et al. This is an open access article distributed under the terms of the Creative Commons Attribution License, which permits unrestricted use, distribution, and reproduction in any medium, provided the original work is properly cited. Data associated with the article are available under the terms of the Creative Commons Zero "No rights reserved" data waiver (CC0 1.0 Public domain dedication).

How to cite this article: Ganapathi $\mathrm{L}$, Arnold $\mathrm{A}$, Jones $\mathrm{S}$ et al. Use of cidofovir in pediatric patients with adenovirus infection [version 2; peer review: 3 approved] F1000Research 2016, 5:758 https://doi.org/10.12688/f1000research.8374.2

First published: 26 Apr 2016, 5:758 https://doi.org/10.12688/f1000research.8374.1 


\section{REVISED Amendments from Version 1}

The new version of the original article clarifies methodology as well as expands on the prior discussion section. In the methods section, we have clarified our center's protocol for testing for adenovirus infection, use of probenecid, how we defined pretreatment creatinine as well as our publication search criteria. We provide a list of concomitant nephrotoxins but omit the analysis as there was insufficient data to account for confounders in this analysis. We have made minor changes to Table 2 as well as updated the literature in Table 3. The discussion section now includes a more involved discussion on the use of cidofovir in solid organ transplant recipients and the overall clinical benefit of cidofovir in general. We have also made brief mention of the use of newer medications such as brincidofovir.

See referee reports

\section{Introduction}

Adenovirus (ADV) is a common cause of respiratory infection in childhood. ADV infections are usually self-limited and asymptomatic in the immunocompetent host but have been recognized as a cause of significant morbidity and mortality in immunocompromised pediatric patients such as recipients of hematopoietic stem cell transplant (HSCT) and solid organ transplant (SOT) ${ }^{1}$. In these patients, ADV is an opportunistic pathogen that may lead to severe localized disease including pneumonia/pneumonitis, hepatitis, hemorrhagic cystitis or disseminated disease with multiorgan failure $^{2-4}$. Case fatality rates in immunocompromised patients with ADV pneumonia have been reported to be as high as $60 \%{ }^{5}$. Currently, there is no FDA-labeled product available for treatment of ADV infection though several agents have been administered for this indication including ribavirin ${ }^{6,7}$, ganciclovir ${ }^{8}$, vidarabine ${ }^{9,10}$, immune globulin ${ }^{11}$ and cidofovir ${ }^{12-21}$.

Cidofovir (CDV), a nucleoside and phosphonate analogue is a broad-spectrum antiviral agent that inhibits viral DNA polymerase and has broad activity in vitro against multiple viruses including all serotypes of $\mathrm{ADV}^{22,23}$. CDV has an FDA indication for the treatment of cytomegalovirus (CMV) retinitis in patients with AIDS. Although this drug does not have an FDA indication for treating $\mathrm{ADV}$, there is evidence of in vivo efficacy of CDV against $\mathrm{ADV}^{12,14}$. While CDV at a standard dose of $5 \mathrm{mg} / \mathrm{kg}$ has been reported as primary therapy for treatment of ADV infection in pediatric and adult hematopoietic stem cell transplantation (HSCT) patients ${ }^{12,21}$, concern exists regarding potential nephrotoxicity. This associated adverse effect has limited the use of CDV for treatment of ADV infections in pediatric patients. To minimize potential toxicity of $\mathrm{CDV}$, modified dosing regimens such as the use of $1 \mathrm{mg} / \mathrm{kg}$ three times weekly have been utilized ${ }^{14}$.

Limited information regarding safety and efficacy of CDV in pediatric patients prompted us to review prior published studies in the literature and conduct a retrospective review of our inpatient use of $\mathrm{CDV}$ at Boston Children's Hospital (BCH).

\section{Methods}

Following Institutional Review Board (IRB) approval (IRBP00015576), a retrospective chart review was conducted for all hospitalized patients at Boston Children's Hospital (BCH), who were prescribed CDV for adenovirus infection from January 2006 through December 2010. Depending on the clinical context, testing for adenoviruses was typically only performed in symptomatic patients, except in the case of stem cell transplant recipients who had a known history of adenovirus infection prior to transplant in which case adenovirus screening was performed routinely. The following data were collected: (1) demographic information, (2) underlying disease state, (3) type of transplant, (4) duration of cidofovir therapy, (6) serum creatinine ( $\mathrm{SCr}$ ) (baseline, peak during therapy, and level up to 2 weeks post last dose), (7) concomitant nephrotoxins prescribed (acyclovir, amikacin, cyclosporine, foscarnet, gentamicin, liposomal amphotericin B, tacrolimus, tobramycin, vancomycin, and intravenous contrast media), (8) sites of ADV detection by viral direct fluorescent antibody (DFA), nucleic acid test, and/or culture, (9) viral quantitative PCR surveillance in whole blood and other sites of infection (all specimens were tested at least weekly before, during and to two weeks post last dose of CDV to evaluate for changes in viral load with a minimum three serial values being obtained before, during and at end of therapy); (10) symptoms of infection, and clinical course including response to therapy, (11) concomitant reduction of immunosuppression and (12) mortality and cause(s) of mortality. All blood sample testing for adenovirus quantitative PCR in whole blood was performed at the Boston Children's Hospital Virology Laboratory using our laboratory developed test, using components of the Argene adenovirus kit (bioMerieux, Cambridge, MA). The Argene adenovirus kit contains primers and probes selective for a 138 base pair (bp) sequence in the Hexon gene of the adenovirus. Using a 5 ' nuclease assay, viral DNA is detected using the primers and fluorescent probes from the Argene kit by means of real time PCR in a Cepheid SmartCycler (Cepheid, Sunnyvale, CA). Of note, all patients who received cidofovir also received probenecid for renal protection at a standard dose of $1.25 \mathrm{~g} / \mathrm{m}^{2} /$ dose administered 3 hours prior to and 2 hours and 8 hours after completion of each 1-hour CDV infusion. We performed a search of relevant literature up till December 2010. The literature search was conducted using PubMed, PubMed Central and Medline databases. Search terms included "treatment of adenoviral infection", "pediatric", "adenovirus" "lung transplant", "liver transplant", "multi-visceral transplant", "kidney transplant", "stem cell transplant", "immunocompromised", "nephrotoxic", "ribavirin", "ganciclovir" and "vidarabine". As we found larger case series pertaining to use of cidofovir in stem cell transplant recipients, we excluded case reports in this population. However, given the paucity of data in solid organ transplant recipients, we included case reports.

\section{Definitions}

As there is no accepted definition for ADV infection or disease, we adopted definitions used in prior studies ${ }^{13}$. Specifically, definite adenovirus disease as follows: Non-gastrointestinal locations: Symptoms and signs from the appropriate organ combined with histopathological documentation of adenovirus and/or 
adenovirus detection by culture, antigen test, or nucleic acid test from biopsy specimens (liver or lung), BAL fluid, or cerebrospinal fluid and without another identifiable cause; Gastrointestinal location: Symptoms together with detection of adenovirus from biopsy material by culture, antigen test, or nucleic acid test.

Probable adenovirus disease as follows: Gastrointestinal tract: Detection of adenovirus in stool by culture, antigen test, or nucleic acid test together with symptoms; Urinary tract: Symptoms of dysuria or hematuria combined with detection of adenovirus by culture, antigen test, or nucleic acid test without another identifiable cause; and Respiratory tract: Symptoms and signs of pneumonia/pneumonitis combined with detection of adenovirus by culture, antigen test, or nucleic acid test without another identifiable cause.

Asymptomatic adenovirus infection as follows: any detection of adenovirus in an asymptomatic patient from stool, blood, urine, or upper airway specimens by viral culture, antigen tests, or PCR.

Adenoviremia was defined as the detection of $>100$ copies of $\mathrm{ADV} / \mathrm{mL}$ of blood (this being the lower limit of detection of the assay). Viral clearance was defined as an ADV viral load of $<100$ copies in blood by quantitative PCR at the end of therapy. Viral response was defined as decrease in viremia by at least one log-reduction (i.e 10-fold). Clinical resolution was defined as resolution of symptoms and/or signs of infection.

Renal dysfunction was defined as a $\geq 50 \%$ increase in $\mathrm{SCr}$ from baseline during the course of CDV therapy. Baseline creatinine was defined as the most recent serum creatinine value prior to initiation of CDV therapy.

\section{Statistical analysis}

Statistical analyses employed Prism 5 for Windows Version 5.04 (GraphPad Software Inc, CA). The Mann-Whitney test was used to assess risk of renal dysfunction. Trends in adenoviremia including pre-treatment viral load, changes in viral load during therapy, and post-treatment viral load were graphed.

\section{Results}

Dataset 1. Raw data for Figure 1

http://dx.doi.org/10.5256/f1000research.8374.d117321

Adenovirus blood viral load is represented in each column at each particular time in days (before or after onset of cidofovir treatment) for each patient.

Dataset 2. Raw data for Figure 2

http://dx.doi.org/10.5256/f1000research.8374.d117322

Nephrotoxicity - pre, peak and post serum creatinine levels in $\mathrm{mg} / \mathrm{dL}$ are represented in each column for each patient.
From January 1, 2006 to December 31, 2010, a total of 16 pediatric patients received CDV for adenovirus infection at our hospital. These 16 patients received 19 courses (three patients received two separate CDV courses). The standard CDV dose of $5 \mathrm{mg} / \mathrm{kg}$ weekly was used in all courses unless there was concern for renal dysfunction at the start of therapy in which case a dosing regimen of $1 \mathrm{mg} / \mathrm{kg}$ three times a week was used. Patient demographics, primary diagnosis, clinical symptoms and course, and sites of adenovirus detection appear in Table 1. Patient age ranged from $0.75-20$ years (mean 6.5 years). Seven (44\%) patients were male. Underlying primary diagnosis included 8 (50\%) HSCT (1 autologous), 4 (25\%) SOT, $2(12.5 \%)$ leukemia, and $2(6.5 \%)$ defined as other. Duration of CDV therapy ranged from 5-82 days (median 33.5 days).

Of the 19 courses prescribed (Table 1), two courses were prescribed in a patient with definite adenovirus disease of the gastrointestinal tract, 15 courses were prescribed in patients with probable disease and two courses were prescribed in patients with asymptomatic infection. Sixteen courses (84\%) were in patients who had a positive blood ADV PCR either in whole blood only or in combination with positive ADV PCR of sputum, stool, urine, broncho-alveolar lavage (BAL) fluid, pericardial fluid or positive sputum adenoviral DFA sample. Respiratory symptoms were the most common presentation (10 courses, 53\%) of which six courses were prescribed for patients with respiratory symptoms and radiological evidence of pneumonia. Two courses were prescribed in patients who presented with prolonged fevers; four courses were prescribed in patients who had worsening diarrhea and colitis, two of which were biopsy proven adenovirus infection; four courses were prescribed in patients with viral sepsis with or without pneumonia; and two courses were administered in patients with severe hemorrhagic cystitis. One course was prescribed in a patient with asymptomatic respiratory tract infection and one course was prescribed in another patient with asymptomatic gastrointestinal infection.

We further examined the 16 blood-positive courses to assess trends in ADV viral load pre-, during and post- CDV therapy (Figure 1). A quantitative reduction in viral load was seen in 15 blood positive courses $(94 \%)$ with viral clearance achieved in 14 (88\%). Of note, all solid organ transplant recipients treated with CDV also had concomitant decrease in their immunosuppression. A single patient (Patient 6) did not demonstrate viral response to therapy and expired. The majority of adenovirus blood-positive CDV courses $(10 / 16,63 \%)$ were associated with clinical improvement with viral clearance; however this was not the case in four courses. Patients 7, 10, 11 and 16 expired despite demonstrating viral clearance. Patients 6, 7 and 16 had multiple other co-infections. Patients 11 and 16 developed severe hemorrhagic cystitis. Patient 11 experienced significant complications of hemorrhagic cystitis including urinary tract obstruction, renal failure and bladder perforation. Patient 16 also had concomitant BK Polyoma virus detected in the urine.

Each patient's medication profile was assessed to determine the number of additional nephrotoxic agents concomitantly prescribed 
Table 1. Demographics, primary diagnosis, sites of ADV detection, clinical symptoms and course of patients included in the study. The age and gender distribution, primary diagnosis, sites of adenovirus detection, symptoms and clinical course of the patients included in the study are shown.

\begin{tabular}{|c|c|c|c|c|c|}
\hline Pt \# & $\begin{array}{l}\text { Age } \\
\text { (yrs) }\end{array}$ & Gender & Diagnosis & $\begin{array}{l}\text { Site(s) of ADV } \\
\text { detection }\end{array}$ & Clinical symptoms \\
\hline $1 \mathrm{a}$ & 12 & $\mathrm{~F}$ & AML - Mismatched UD Cord SCT & Sp, S, R & Pneumonia \\
\hline $1 b$ & 12 & $\mathrm{~F}$ & AML - Mismatched UD Cord SCT & $\mathrm{Sp}$ & Fever and Respiratory symptoms \\
\hline 2 & 12 & M & $\begin{array}{c}\text { Severe Idiosyncratic Immunodeficiency } \\
- \text { MRD SCT }\end{array}$ & $\mathrm{B}, \mathrm{S}$ & Prolonged fever \\
\hline 3 & 3 & M & AML - Chemotherapy & $\mathrm{B}, \mathrm{S}, \mathrm{U}$ & Pneumonia \\
\hline 4 & 4 & $\mathrm{~F}$ & Neuroblastoma - Autologous SCT & $B, S, P F$ & Prolonged fever, pericardial effusion \\
\hline 5 & 19 & $\mathrm{~F}$ & Cystic Fibrosis - Lung Transplant & $\mathrm{B}, \mathrm{BAL}$ & Asymptomatic \\
\hline${ }^{*} 6$ & 1 & $\mathrm{~F}$ & Familial HLH - MUD SCT & $\mathrm{B}, \mathrm{Sp}$ & $\begin{array}{c}\text { Pneumonia, sepsis. Other } \\
\text { co-infections including Enterococcus } \\
\text { bacteremia, EBV viremia }\end{array}$ \\
\hline *7 & 0.83 & M & $\begin{array}{l}\text { Persistent pulmonary hypertension, } \\
\text { cardiomyopathy }\end{array}$ & B & $\begin{array}{l}\text { Fever, sepsis, pneumonia. Other } \\
\text { co-infections including } \\
\text { stenotrophomonas bacteremia }\end{array}$ \\
\hline $8 a$ & 20 & M & ALL - MRD SCT and MUD SCT & $\mathrm{BAL}, \mathrm{B}, \mathrm{U}$ & Pneumonia \\
\hline $8 b$ & 20 & M & ALL - MRD SCT and MUD SCT & S & Asymptomatic \\
\hline 9 & 1 & $\mathrm{~F}$ & $\begin{array}{l}\text { Congenital Nephrotic Syndrome \& } \\
\text { Hepatoblastoma - Combined Liver \& } \\
\text { Kidney Transplant }\end{array}$ & $\mathrm{B}, \mathrm{Sp}$ & Respiratory symptoms \\
\hline *10 & 0.75 & M & HLH - SCT & $S, B, U$ & Fever, sepsis, diarrhea \\
\hline *11 & 5 & $\mathrm{~F}$ & $\begin{array}{l}\text { CID and Lymphoproliferative Disorder } \\
\text { - Mismatched UD Cord SCT }\end{array}$ & B & $\begin{array}{c}\text { Fever, respiratory symptoms, diarrhea, } \\
\text { hemorrhagic cystitis }\end{array}$ \\
\hline 12 & 2 & $\mathrm{~F}$ & $\begin{array}{c}\text { Congenital Cardiac Defect - Heart } \\
\text { Transplant }\end{array}$ & B & Fever, sepsis \\
\hline 13 & 2 & $\mathrm{~F}$ & ALL - Chemotherapy & $B, S$ & Fever, respiratory symptoms \\
\hline 14 & 2 & $\mathrm{~F}$ & Cerebral Palsy & B & Pneumonia \\
\hline $15 a$ & 1.8 & M & $\begin{array}{c}\text { Hepatoblastoma - Multivisceral } \\
\text { Transplant }\end{array}$ & $B, S$ & $\begin{array}{l}\text { Increased stoma output, rejection, } \\
\text { biopsy proven ADV In stoma mucosa }\end{array}$ \\
\hline $15 b$ & 1.8 & M & Hepatoblastoma - Multiviseral Transplant & $B, S$ & Increased stoma output \\
\hline *16 & 17 & M & AML - MUD SCT & $B, U$ & $\begin{array}{c}\text { Hemorrhagic cystitis. Other } \\
\text { co-infections including BK viruria and } \\
\text { EBV viremia }\end{array}$ \\
\hline
\end{tabular}

ALL, acute lymphoblastic leukemia; AML, acute myeloid leukemia; CID, congenital immunodeficiency; CML, chronic myelogenous leukemia; HLH, hemophagocytic lymphohistiocytosis; MRD, matched related donor; MUD, matched unrelated donor; Pt \#, patient number; SCID, severe combined immunodeficiency disorder; SCT, stem cell transplant; UD, unrelated donor; Yrs, years; Site of adenovirus detection: S, stool, Sp, sputum, B, blood, BAL, bronchoalveolar lavage, R, respiratory DFA, CSF, cerebrospinal fluid, U, Urine, PF, Pericardial Fluid; *indicates patient expired

during CDV therapy (from Day 1 to 7 days post last CDV dose) (Table 2).

Administration of CDV was significantly associated with occurrence of renal dysfunction when comparing the peak $\mathrm{Cr}$ measured during CDV therapy to the baseline serum $\mathrm{Cr}(\mathrm{p}=0.0016)$. Eleven courses (58\%) were associated with development of renal dysfunction. $\mathrm{Cr}$ increased by a mean of $\sim 50 \%$ from baseline during CDV therapy.. Of the courses with elevation in serum creatinine, $64 \%$ demonstrated return to pre-treatment creatinine levels following cessation of CDV therapy.

\section{Discussion}

In this retrospective review of patients treated with CDV for adenovirus infection at our hospital during a 5-year period, we assessed the safety and potential efficacy of the medication in pediatric patients. Our review yielded a case series of 16 patients. While the number of patients is modest, this series adds to the existing 


\section{Viremia}

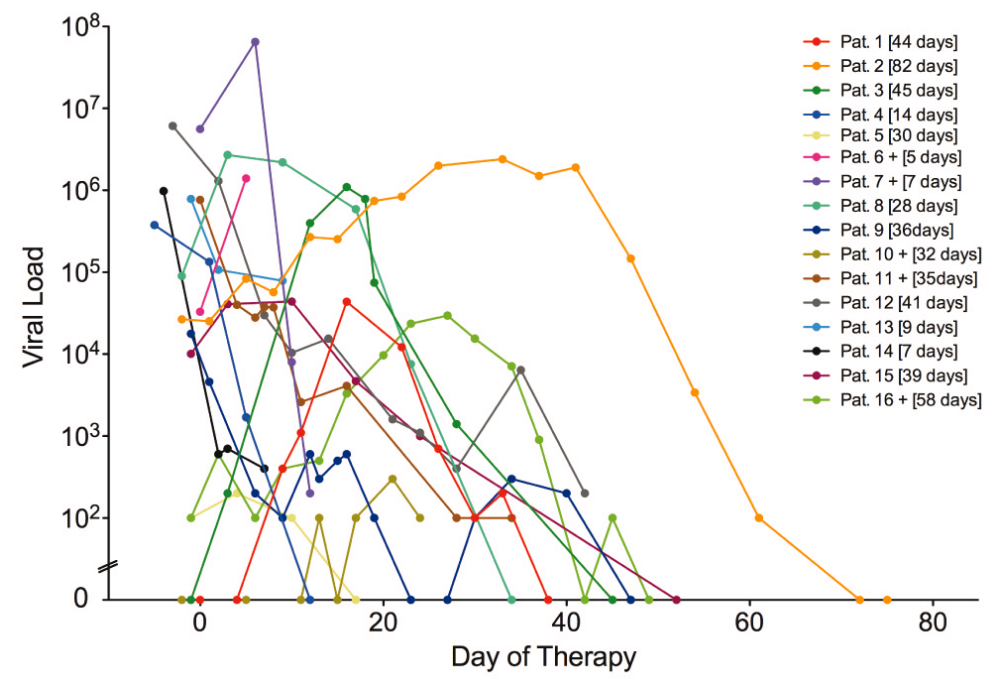

Figure 1. Kinetics of Adenovirus blood viral load under cidofovir treatment. Viral loads of 16 patients with quantitative blood adenovirus PCR treated with cidofovir, are shown. Day of therapy $\leq 0$ denotes pre-treatment viral loads. Up to two post-treatment values are shown where available and informative. Each patient's individual treatment duration is shown in the legend. + denotes patient expired.

Table 2. Additional nephrotoxic agents prescribed and changes in serum creatinine during cidofovir therapy. Absolute values for serum creatinine in $\mathrm{mg} / \mathrm{dL}$ for each patient are represented pre-treatment, during treatment (peak serum creatinine), and post-treatment. Additional nephrotoxic agents that each patient received are also represented.

\begin{tabular}{|c|c|c|c|c|}
\hline Pt \# & $\begin{array}{l}\text { Pre-treatment } \\
\text { Serum } \mathrm{Cr}\end{array}$ & $\begin{array}{l}\text { Peak Serum } \\
\mathrm{Cr}\end{array}$ & $\begin{array}{l}\text { Post-treatment } \\
\text { Serum } \mathrm{Cr}\end{array}$ & Additional nephrotoxic agents \\
\hline $1 \mathrm{a}$ & 0.3 & 1.2 & 0.6 & ambisome, cyclosporine, gentamicin \\
\hline $1 b$ & 0.6 & 1 & 0.6 & cyclosporine \\
\hline 2 & 0.6 & 0.6 & 0.5 & cyclosporine, ganciclovir, pentamidine \\
\hline 3 & 0.3 & 0.6 & 0.2 & vancomycin, ambisome, gentamicin \\
\hline 4 & 0.3 & 0.3 & 0.2 & acyclovir \\
\hline 5 & 0.4 & 0.7 & 0.5 & tacrolimus \\
\hline 6 & 0.2 & 0.4 & 0.6 & ambisome, ganciclovir, pentamidine, tacrolimus, vancomycin \\
\hline 7 & 0.2 & 0.5 & 0.7 & gentamicin, \\
\hline $8 a$ & 1 & 1.7 & 0.4 & ambisome, vancomycin \\
\hline $8 b$ & 0.4 & 2.6 & 1.3 & ganciclovir, tacrolimus, vancomycin \\
\hline 9 & 0.3 & 2.7 & 0.6 & chemotherapy, immunosuppression \\
\hline 10 & 0.2 & 0.2 & 0.2 & acyclovir, ambisome, cyclosporine, vancomycin \\
\hline 11 & 0.4 & 1.6 & 0.6 & $\begin{array}{c}\text { gentamicin, acyclovir, ambisome, cyclosporine, foscarnet, } \\
\text { ganciclovir }\end{array}$ \\
\hline 12 & 0.5 & 1.6 & 0.6 & gentamicin, ganciclovir, tacrolimus \\
\hline 13 & 0.2 & 0.2 & 0.2 & ambisome,foscarnet, ganciclovir \\
\hline 14 & 0.2 & 0.2 & 0.2 & vancomycin \\
\hline $15 a$ & 0.3 & 0.3 & 0.3 & tacrolimus, vancomycin \\
\hline $15 b$ & 0.2 & 0.3 & 0.2 & tacrolimus, vancomycin \\
\hline 16 & 0.3 & 0.5 & 0.3 & cyclosporine, gentamicin, vancomycin, contrast \\
\hline
\end{tabular}

Pt \#, patient number; Cr, creatinine; all creatinine values are in mg/dL. 
literature describing the use of $\mathrm{CDV}$ in pediatric recipients of HSCT, SOT and chemotherapy for oncologic diagnoses (Table 3).

Similar to other studies the majority of our patients had received a HSCT or had an oncologic diagnosis and received chemotherapy. We identified eight publications describing the use of CDV for adenovirus infection in the setting of HSCT or oncologic diagnoses treated with chemotherapy (Table 3). Three of these studies ${ }^{14,17,27}$ reported viral clearance in $89-100 \%$ of their patients. We observed similar rates of viral clearance $(88 \%)$ but this was not consistently associated with clinical improvement.
There are very few reports on the use of CDV for adenovirus infection in pediatric SOT recipients, which have largely been restricted to reports of children receiving liver or lung transplants ${ }^{16,20,24,25,29,30}$. We identified six publications reporting the use of CDV for adenovirus infection in pediatric SOT recipients limited to one to four per report with the majority of these children having received liver or lung transplants ${ }^{16,20,24,25,29,30}$. Doan et al. ${ }^{25}$ described children who had received lung transplants with reported viral clearance in three of their four patients. Our case series contributes patients who received several types of SOT including lung, heart, combined kidney and liver, and multi-visceral transplants. All patients with

Table 3. Summary of studies describing use of cidofovir in pediatric HSCT and SOT recipient. Published studies in the literature describing use of cidofovir in pediatric HSCT and SOT recipients are summarized. Toxicities, and clinical outcomes reported in each study are highlighted.

\begin{tabular}{|c|c|c|c|c|c|c|c|}
\hline Study & $\begin{array}{l}\text { \# Patients } \\
\text { (N) }\end{array}$ & Clinical Setting & $\begin{array}{l}\text { Median } \\
\text { Age }\end{array}$ & Cidofovir dosage & $\begin{array}{l}\text { Duration } \\
\text { of } \\
\text { cidofovir } \\
\text { use }\end{array}$ & $\begin{array}{l}\text { Potential } \\
\text { Toxicity }\end{array}$ & Outcome \\
\hline Hoffman $^{14}$ & 8 & HSCT & $7 \mathrm{yrs}$ & $\begin{array}{l}1 \mathrm{mg} / \mathrm{kg} / \mathrm{dose} \\
\text { three times weekly } \\
\times 9 \text { doses or until } \\
\text { clearance }\end{array}$ & $\begin{array}{l}3 \text { weeks-8 } \\
\text { months }\end{array}$ & $\begin{array}{l}\text { Well tolerated; } \\
\text { no toxicity } \\
\text { reported }\end{array}$ & $\begin{array}{l}100 \% \text { viral } \\
\text { suppression } \\
3 \text { recurrences } \\
4 \text { expired ( } 2 \\
\text { ADV-related) }\end{array}$ \\
\hline Muller ${ }^{12}$ & 10 & $\mathrm{HSCT}$ & Not reported & $\begin{array}{l}5 \mathrm{mg} / \mathrm{kg} / \mathrm{dose} \\
\text { weekly up to } 6 \\
\text { weeks, then every } \\
\text { other week for } 3 \\
\text { more doses }\end{array}$ & $\begin{array}{l}3 \text { weeks-6 } \\
\text { months }\end{array}$ & $\begin{array}{l}30 \% \\
\text { nephrotoxicity } \\
\text { (50\% increase } \\
\text { Cr) }\end{array}$ & $\begin{array}{l}9 \text { virologic } \\
\text { clearance } \\
5 \text { recurrences } \\
1 \text { expired } \\
\text { (interstitial } \\
\text { pneumonitis) }\end{array}$ \\
\hline Anderson $^{19}$ & 7 & HSCT & $1.5 \mathrm{yrs}$ & $\begin{array}{l}\text { Preemptive } \\
\text { therapy: } 1 \mathrm{mg} / \mathrm{kg} / \\
\text { dose three times } \\
\text { weekly } \times 3 \text { weeks }\end{array}$ & 3 weeks & $\begin{array}{l}\text { Well tolerated } \\
\text { without } \\
\text { significant } \\
\text { toxicity reported }\end{array}$ & $\begin{array}{l}\text { No patient } \\
\text { developed ADV } \\
\text { viremia } \\
2 \text { expired (non- } \\
\text { ADV related) }\end{array}$ \\
\hline Bhadri ${ }^{15}$ & 23 & $\begin{array}{l}87 \% \text { HSCT } \\
13 \% \text { oncologic } \\
\text { receiving } \\
\text { chemotherapy }\end{array}$ & $5.7 \mathrm{yrs}$ & $\begin{array}{l}5 \mathrm{mg} / \mathrm{kg} / \mathrm{dose} \\
\text { weekly, } 3 \mathrm{mg} / \mathrm{kg} / \\
\text { dose weekly or } 1 \\
\mathrm{mg} / \mathrm{kg} / \text { dose three } \\
\text { times weekly }\end{array}$ & $\begin{array}{l}\text { Median } \\
6 \text { weeks } \\
\text { (1-26 } \\
\text { weeks) }\end{array}$ & $\begin{array}{l}9 \% \text { Grade } 1 \\
\text { nephrotoxicity } \\
\text { defined by } \\
\text { increase of } \\
\text { creatinine up to } \\
1.5 \text { times upper } \\
\text { limit of normal }\end{array}$ & $\begin{array}{l}85 \% \text { of } 20 \\
\text { evaluable } \\
\text { patients } \\
\text { considered } \\
\text { successful } \\
\text { by Ljungman } \\
\text { criteria }^{13} \\
17 \text { expired }\end{array}$ \\
\hline Yusuf ${ }^{17}$ & 57 & $\begin{array}{l}90 \% \text { HSCT } \\
10 \% \text { oncologic } \\
\text { receiving } \\
\text { chemotherapy }\end{array}$ & 8 yrs & $\begin{array}{l}5 \mathrm{mg} / \mathrm{kg} / \mathrm{dose} \\
\text { weekly } \times 2 \text { weeks, } \\
\text { then every other } \\
\text { week until } 3 \\
\text { negative ADV } \\
\text { samples }\end{array}$ & $\begin{array}{l}\text { Median } \\
60 \text { days } \\
\text { (1 week-9 } \\
\text { months) }\end{array}$ & $\begin{array}{l}\text { No toxicity } \\
\text { reported }\end{array}$ & $\begin{array}{l}98 \% \text { successful } \\
\text { viral clearance } \\
14 \% \text { recurrence } \\
29 \text { expired (1 } \\
\text { ADV-related } \\
\text { death) }\end{array}$ \\
\hline Legrand $^{18}$ & 7 & $\mathrm{HSCT}$ & $6.4 \mathrm{yrs}$ & $\begin{array}{l}5 \mathrm{mg} / \mathrm{kg} / \mathrm{dose} \\
\text { weekly } \times 3 \text { wks } \\
\text { then every other } \\
\text { wk or } 10 \text { days }\end{array}$ & $\begin{array}{l}25-330 \\
\text { days }\end{array}$ & $\begin{array}{l}43 \% \\
\text { nephrotoxicity }\end{array}$ & $\begin{array}{l}71 \% \text { deemed } \\
\text { recovered } \\
2 \text { expired ( } 1 \\
\text { ADV related } \\
\text { death) }\end{array}$ \\
\hline
\end{tabular}




\begin{tabular}{|c|c|c|c|c|c|c|c|}
\hline Study & $\begin{array}{l}\text { \# Patients } \\
\text { (N) }\end{array}$ & Clinical Setting & $\begin{array}{l}\text { Median } \\
\text { Age }\end{array}$ & Cidofovir dosage & $\begin{array}{l}\text { Duration } \\
\text { of } \\
\text { cidofovir } \\
\text { use }\end{array}$ & $\begin{array}{l}\text { Potential } \\
\text { Toxicity }\end{array}$ & Outcome \\
\hline Sivaprakasam ${ }^{26}$ & 8 & HSCT & $11 \mathrm{yrs}$ & $\begin{array}{l}1 \mathrm{mg} / \mathrm{kg} / \text { dose } \\
3 \text { times weekly } \\
\text { (4 patients also } \\
\text { received IV } \\
\text { ribavirin } \\
5 \mathrm{mg} / \mathrm{kg} 3 \text { times } \\
\text { daily) }\end{array}$ & $\begin{array}{l}\text { Not } \\
\text { reported }\end{array}$ & $\begin{array}{l}2 \text { cases marrow } \\
\text { failure, } 1 \text { case } \\
\text { nephropathy }\end{array}$ & $\begin{array}{l}3 \text { expired } \\
\text { (attributed } \\
\text { to ADV and } \\
\text { GVHD) }\end{array}$ \\
\hline Williams $^{27}$ & 9 & HSCT & 3 yrs & $\begin{array}{l}5 \mathrm{mg} / \mathrm{kg} / \mathrm{dose} \\
\text { once weekly } \\
\text { until } 3 \text { weeks of } \\
\text { negative results or } \\
\text { pt no longer high } \\
\text { risk; if underlying } \\
\text { renal dysfunction } \\
1 \mathrm{mg} / \mathrm{kg} / \text { dose } 3 \\
\text { times weekly }\end{array}$ & $\begin{array}{l}\text { Median } \\
8 \text { doses } \\
\text { (3-32 } \\
\text { doses) }\end{array}$ & $\begin{array}{l}22 \% \text { renal } \\
\text { failure } \\
\text { (compared to } \\
80 \% \text { untreated } \\
\text { comparator } \\
\text { group) }\end{array}$ & $\begin{array}{l}89 \% \text { ADV } \\
\text { clearance } \\
3 \text { expired (1 } \\
\text { ADV related) }\end{array}$ \\
\hline Engelmann ${ }^{16}$ & 1 & Liver transplant & 7 months & $\begin{array}{l}6 \mathrm{mg} / \mathrm{kg} / \text { dose } \times 1 \\
\text { with } 1 \text { repeat dose } \\
6 \text { days later }\end{array}$ & 2 weeks & $\begin{array}{l}\text { No toxicity } \\
\text { reported }\end{array}$ & $\begin{array}{l}\text { Liver rejection; } \\
\text { reported to } \\
\text { have slow } \\
\text { recovery }\end{array}$ \\
\hline Wallot ${ }^{20}$ & 2 & Liver transplant & $\begin{array}{l}8 \text { months and } \\
14 \text { months }\end{array}$ & $\begin{array}{l}1 \mathrm{mg} / \mathrm{kg} / \mathrm{dose} \\
\text { three times weekly }\end{array}$ & 5-8 weeks & $\begin{array}{l}1 \text { moderate } \\
\text { neutropenia, } 1 \\
\text { transient rise in } \\
\text { creatinine }\end{array}$ & $\begin{array}{l}\text { Blood PCR ADV } \\
\text { clearance in } \\
\text { both patients } \\
\text { No deaths }\end{array}$ \\
\hline Carter ${ }^{24}$ & 1 & Liver transplant & 7 months & $\begin{array}{l}1 \mathrm{mg} / \mathrm{kg} / \mathrm{dose} \\
\text { three times weekly }\end{array}$ & 7 weeks & $\begin{array}{l}\text { Transient } \\
\text { acidosis and } \\
\text { proteinuria }\end{array}$ & $\begin{array}{l}\text { ADV viral } \\
\text { culture and } \\
\text { blood PCR } \\
\text { became } \\
\text { negative }\end{array}$ \\
\hline Doan $^{25}$ & 4 & Lung transplant & $<3$ yrs & $\begin{array}{l}1 \mathrm{mg} / \mathrm{kg} / \mathrm{dose} \\
\text { every other day to } \\
\text { three times weekly } \\
\text { plus IVIG } \\
\text { ( } 1 \mathrm{pt} \text { increased } \\
\text { dose to } 2 \mathrm{mg} / \mathrm{kg} / \\
\text { dose; } \\
1 \mathrm{pt} \text { increased } \\
\text { frequency to } \\
\text { daily therapy } \times 2 \\
\text { weeks) }\end{array}$ & 4 weeks & $\begin{array}{l}\text { No toxicity } \\
\text { reported }\end{array}$ & $\begin{array}{l}75 \% \text { negative } \\
\text { blood ADV PCR } \\
1 \text { death }\end{array}$ \\
\hline Siedemann ${ }^{29}$ & 6 & $\begin{array}{c}\text { 70\% Liver } \\
\text { transplant } \\
15 \% \text { HSCT } \\
15 \% \text { combined } \\
\text { liver and HSCT }\end{array}$ & $2 \mathrm{yrs}$ & $\begin{array}{l}5 \mathrm{mg} / \mathrm{kg} / \mathrm{dose} \\
\text { once weekly }\end{array}$ & $\begin{array}{l}\text { Not } \\
\text { reported }\end{array}$ & Not reported & $\begin{array}{l}3 \text { expired (all } \\
\text { ADV related) }\end{array}$ \\
\hline Leurez-Ville ${ }^{30}$ & $\begin{array}{c}37 \\
\text { (8 patients } \\
\text { with } \\
\text { disseminated } \\
\text { infection) }\end{array}$ & $\begin{array}{c}60 \% \text { HSCT } \\
20 \% \text { solid organ } \\
\text { transplant (1 liver, } \\
2 \text { small bowel, } 5 \\
\text { combined liver } \\
\text { and small bowel) } \\
20 \% \text { congenital } \\
\text { immunodeficiency }\end{array}$ & $\begin{array}{l}2.2 \text { yrs (only } \\
\text { cases of } \\
\text { disseminated } \\
\text { infection } \\
\text { reported) }\end{array}$ & $\begin{array}{l}5 \mathrm{mg} / \mathrm{kg} / \mathrm{dose} \\
\text { once weekly } \\
\text { for two weeks } \\
\text { followed by } \\
5 \mathrm{mg} / \mathrm{kg} \text { every } 2 \\
\text { weeks or } 10 \text { days; } \\
\text { only patients with } \\
\text { disseminated } \\
\text { infection received } \\
\text { cidofovir) }\end{array}$ & $\begin{array}{l}\text { Not } \\
\text { reported }\end{array}$ & $\begin{array}{l}1 \text { patient with } \\
\text { persistent } \\
\text { nephroxicity } \\
\text { requiring dose } \\
\text { adjustment of } \\
\text { cidofovir }\end{array}$ & $\begin{array}{l}60 \% \text { with } \\
\text { disseminated } \\
\text { infection } \\
\text { recovered } \\
3 \text { patients with } \\
\text { disseminated } \\
\text { infection } \\
\text { expired }\end{array}$ \\
\hline
\end{tabular}


SOT in our series demonstrated viral clearance as well as resolution of symptoms, which may have reflected a combination of both the antiviral effect of CDV coupled with reduced immunosuppression. Interestingly, a prospective study performed in adult liver, kidney and heart transplant recipients noted the transient nature of adenoviremia and self limited infection in this population even in the absence of any treatment or interventions ${ }^{28}$. However, the authors also note that the majority of patients in whom adenoviremia was detected in their study were asymptomatic or only mildly symptomatic, and thus differed from patients with severe infections who are more frequently reported in the pediatric transplant population and in lung allograft recipients. In contrast, Seidemann and colleagues ${ }^{29}$ report mortality in 2 of 5 pediatric solid organ transplant recipients despite the use of cidofovir in their case series. Similarly, Leurez-Ville et al. ${ }^{30}$ report mortality in 1 of 3 pediatric solid organ transplant recipients with disseminated adenovirus infection. The patients who expired in both these reports had severe symptoms and ultimately died of septic multiorgan failure. With the exception of a lung transplant recipient, all other solid organ transplant recipients with adenovirus infection in our study were more than mildly symptomatic. While other reports suggest the possibility of self-resolution of adenoviremia and absence of significant clinical sequelae in asymptomatic or mildly symptomatic solid organ transplant recipients, the propensity for serious infections and mortality has also been highlighted in the pediatric specific solid organ transplant literature ${ }^{29,30}$.

Two-thirds of our patients experienced resolution of their symptoms and had an overall favorable clinical course with recovery. However, one-third died all of whom were stem cell transplant recipients. With the exception of one patient it is unclear whether adenovirus was the direct cause of mortality in these patients. Our observations are consistent with what has been reported in the literature pertaining to outcomes in stem cell transplant recipients with adenovirus infections who have been treated with $\mathrm{CDV}^{9,12,14,18,23,27}$. Among SCT patients mortality remains high (10\%-70\%) even when clearance from blood is seen. It is hence difficult to conclude from these data whether or not cidofovir provided any clinical benefit. It should also be noted that two patients in our series received cidofovir despite being asymptomatic. One such patient was a lung transplant recipient in whom adenovirus was detected in both blood and BAL fluid, the other was a stem cell transplant recipient with a prior history of recurrent viremia and pneumonia. Both these patients were considered to be at high risk for complications from adenovirus infection thus leading to the decision to use cidofovir.

In our case series, renal dysfunction was common during CDV therapy with patients experiencing an average $50 \%$ increase of serum creatinine from their baseline. However, renal dysfunction was transient in the majority of patients with serum creatinine returning to baseline after cessation of CDV therapy. While some studies have reported no toxicities related to the use of $\mathrm{CDV}^{14,16,17,19,25}$, the transient nature of nephrotoxicity observed has been reported by other studies ${ }^{20,24}$. Finally, brincidofovir (CMX001), an orally administered lipid -conjugate derivative of cidofovir is currently being investigated in clinical trials and is reported to have no nephrotoxicity ${ }^{31}$.

Our study has several limitations. Most notably, the small number of patients precluded evaluation of other factors that may impact infection resolution such as immunosuppressive regimens, and additional factors that may impact degree of renal dysfunction. Nevertheless, our study adds to the limited reported literature of pediatric ADV patients treated with CDV.

\section{Data availability \\ F1000Research: Dataset 1. Raw data for Figure 1, 10.5256/ f1000research.8374.d117321 ${ }^{32}$}

F1000Research: Dataset 2. Raw data for Figure 2, 10.5256/ f1000research.8374.d117322 33

\section{Author contributions}

OL conceived the study. LG, AA and SJ collected data and prepared the first draft of the manuscript. LG, AP and DG performed data analysis. $\mathrm{MH}$ contributed to the preparation of the manuscript. All authors were involved in the revision of the draft manuscript and have agreed to the final content.

\section{Competing interests}

No competing interests were disclosed.

\section{Grant information}

OL's laboratory is supported by a Boston Children's Hospital Department of Medicine award to the Precision Vaccines Program as well as Global Health (OPPGH5284) and Grand Challenges Explorations (OPP1035192) awards from the Bill \& Melinda Gates Foundation and by NIH grants 1R01AI10013501 and 3R01AI067353-05S1 and National Institute of Allergy \& Infectious Diseases Adjuvant Discovery Program, Contract No. HHSN272201400052C. The Levy Laboratory has received sponsored research support from VentiRx Pharmaceuticals, 3M Drug Delivery Systems, MedImmune, and Crucell (Johnson \& Johnson)companies that develop adjuvants and vaccines.

The funders had no role in study design, data collection and analysis, decision to publish, or preparation of the manuscript. 
1. Ison MG: Adenovirus infections in transplant recipients. Clin Infect Dis. 2006; 43(3): 331-339.

PubMed Abstract | Publisher Full Text

2. Carrigan DR: Adenovirus infections in immunocompromised patients. Am J Med. 1997; 102(3A): 71-74.

PubMed Abstract

3. Howard DS, Phillips II GL, Reece DE, et al:: Adenovirus infections in hematopoietic stem cell transplant recipients. Clin Infect Dis. 1999; 29(6): 1494-1501.

PubMed Abstract | Publisher Full Tex

4. Hale GA, Heslop HE, Krance RA, et al:: Adenovirus infection after pediatric bone marrow transplantation. Bone Marrow Transplant. 1999; 23(3): 277-282. PubMed Abstract | Publisher Full Text

5. Hierholzer JC: Adenoviruses in the immunocompromised host. Clin Microbio Rev. 1992; 5(3): 262-274.

PubMled Abstract | Publisher Full Text | Free Full Text

6. Gavin PJ, Katz BZ: Intravenous ribavirin treatment for severe adenovirus disease in immunocompromised children. Pediatrics. 2002; 110(1 Pt 1): e9. PubMed Abstract

7. McCarthy AJ, Bergin M, De Silva LM, et al:: Intravenous ribavirin therapy for disseminated adenovirus infection. Pediatr Infect Dis J. 1995; 14(11): 1003-1004.

PubMed Abstract | Publisher Full Tex

8. Chen FE, Liang RH, Lo JY, et al:: Treatment of adenovirus-associated haemorrhagic cystitis with ganciclovir. Bone Marrow Transplant. 1997; 20(11): 997-999.

PubMed Abstract | Publisher Full Text

9. Kawakami M, Ueda S, Maeda T, et al:: Vidarabine therapy for virus-associated cystitis after allogeneic bone marrow transplantation. Bone Marrow Transplant. 1997; 20(6): 485-490.

PubMed Abstract | Publisher Full Text

10. Kitabayashi A, Hirokawa M, Kuroki J, et al.: Successful vidarabine therapy for adenovirus type 11-associated acute hemorrhagic cystitis after allogeneic bone marrow transplantation. Bone Marrow Transplant. 1994; 14(5): 853-854. PubMed Abstract

11. Saquib R, Melton LB, Chandrakantan A, et al.: Disseminated adenovirus infection in renal transplant recipients: the role of cidofovir and intravenous immunoglobulin. Transpl Infect Dis. 2010; 12(1): 77-83. PubMed Abstract | Publisher Full Text

12. Muller WJ, Levin MJ, Shin YK, et al:: Clinical and in vitro evaluation of cidofovir for treatment of adenovirus infection in pediatric hematopoietic stem cell transplant recipients. Clin Infect Dis. 2005; 41(12): 1812-1816. PubMed Abstract | Publisher Full Text

13. Ljungman $\mathrm{P}$, Ribaud $\mathrm{P}$, Eyrich $\mathrm{M}$, et al.: Cidofovir for adenovirus infections after allogeneic hematopoietic stem cell transplantation: a survey by the Infectious Diseases Working Party of the European Group for Blood and Marrow Transplantation. Bone Marrow Transplant. 2003; 31(6): 481-486. PubMed Abstract | Publisher Full Text

14. Hoffman JA, Shah AJ, Ross LA, et al:: Adenoviral infections and a prospective trial of cidofovir in pediatric hematopoietic stem cell transplantation. Biol Blood Marrow Transplant. 2001; 7(7): 388-394. PubMed Abstract | Publisher Full Text

15. Bhadri VA, Lee-Horn L, Shaw PJ: Safety and tolerability of cidofovir in high-risk pediatric patients. Transpl Infect Dis. 2009; 11(4): 373-379. PubMed Abstract | Publisher Full Text

16. Engelmann G, Heim A, Greil J, et al.: Adenovirus infection and treatment with cidofovir in children after liver transplantation. Pediatr Transplant. 2009; 13(4): 421-428.

PubMed Abstract | Publisher Full Text

17. Yusuf U, Hale GA, Carr J, et al:: Cidofovir for the treatment of adenoviral infection in pediatric hematopoietic stem cell transplant patients. Transplantation. 2006; 81(10): 1398-1404. PubMed Abstract | Publisher Full Tex
18. Legrand F, Berrebi D, Houhou N, et al.: Early diagnosis of adenovirus infection and treatment with cidofovir after bone marrow transplantation in children. Bone Marrow Transplant. 2001; 27(6): 621-626.

PubMed Abstract | Publisher Full Text

19. Anderson EJ, Guzman-Cottrill JA, Kletzel M, et al:: High-risk adenovirus-infected pediatric allogeneic hematopoietic progenitor cell transplant recipients and preemptive cidofovir therapy. Pediatr Transplant. 2008; 12(2): 219-227. PubMed Abstract | Publisher Full Text

20. Wallot MA, Dohna-Schwake C, Auth M, et al.: Disseminated adenovirus infection with respiratory failure in pediatric liver transplant recipients: impact of intravenous cidofovir and inhaled nitric oxide. Pediatr Transplant. 2006; 10(1): 121-127.

PubMed Abstract | Publisher Full Text

21. Ribaud P, Scieux C, Freymuth F, et al:: Successful treatment of adenovirus disease with intravenous cidofovir in an unrelated stem-cell transplant recipient. Clin Infect Dis. 1999; 28(3): 690-691.

PubMed Abstract | Publisher Full Text

22. Naesens L, Lenaerts L, Andrei G, et al.: Antiadenovirus activities of several classes of nucleoside and nucleotide analogues. Antimicrob Agents Chemother. 2005; 49(3): 1010-1016. PubMed Abstract | Publisher Full Text | Free Full Text

23. Morfin F, Dupuis-Girod S, Mundweiler S, et al.: In vitro susceptibility of adenovirus to antiviral drugs is species-dependent. Antivir Ther. 2005; 10(2): 225-229. PubMed Abstract

24. Carter BA, Karpen SJ, Quiros-Tejeira RE, et al:: Intravenous Cidofovir therapy for disseminated adenovirus in a pediatric liver transplant recipient. Transplantation. 2002; 74(7): 1050-1052.

PubMed Abstract

25. Doan ML, Mallory GB, Kaplan SL, et al.: Treatment of adenovirus pneumonia with cidofovir in pediatric lung transplant recipients. J Heart Lung Transplant. 2007; 26(9): 883-889.

PubMed Abstract | Publisher Full Text

26. Sivaprakasam $\mathrm{P}$, Carr TF, Coussons $\mathrm{M}$, et al: Improved outcome from invasive adenovirus infection in pediatric patients after hemopoietic stem cell transplantation using intensive clinical surveillance and early intervention. $J$ Pediatr Hematol Oncol. 2007; 29(2): 81-85.

PubMed Abstract | Publisher Full Text

27. Williams KM, Agwu AL, Dabb AA, et al:: A clinical algorithm identifies high risk pediatric oncology and bone marrow transplant patients likely to benefit from treatment of adenoviral infection. J Pediatr Hematol Oncol. 2009; 31(11) 825-831.

PubMed Abstract | Publisher Full Text | Free Full Text

28. Humar A, Kumar D, Mazzulli T, et al:: A surveillance study of adenovirus infection in adult solid organ transplant recipients. Am J Transplant. 2005 5(10): 2555-9.

PubMed Abstract | Publisher Full Text

29. Seidemann K, Heim A, Pfister ED, et al:: Monitoring of adenovirus infection in pediatric transplant recipients by quantitative PCR: report of six cases and review of the literature. Am J Transplant. 2004; 4(12): 2102-8. PubMed Abstract | Publisher Full Text

30. Leruez-Ville M, Minard V, Lacaille F, et al:: Real-time blood plasma polymerase chain reaction for management of disseminated adenovirus infection. Clin Infect Dis. 2004; 38(1): 45-52.

PubMed Abstract | Publisher Full Text

31. Wold WS, Toth K: New drug on the horizon for treating adenovirus. Expert Opin Pharmacother. 2015; 16(14): 2095-9. PubMed Abstract | Publisher Full Text | Free Full Text

32. Ganapathi L, Arnold A, Jones S, et al.: Dataset 1 in: Use of cidofovir in pediatric patients with adenovirus infection. F1000Research. 2016. Data Source

33. Ganapathi L, Arnold A, Jones S, et al.: Dataset 2 in: Use of cidofovir in pediatric patients with adenovirus infection. F1000Research. 2016.

Data Source 


\section{Open Peer Review}

\section{Current Peer Review Status:}

\section{Version 2}

Reviewer Report 04 January 2017

https://doi.org/10.5256/f1000research.11225.r18581

(C) 2017 Hunstad D. This is an open access peer review report distributed under the terms of the Creative Commons Attribution License, which permits unrestricted use, distribution, and reproduction in any medium, provided the original work is properly cited.

\section{David A Hunstad}

Division of Pediatric Infectious Diseases, Washington University in St Louis, St Louis, MO, USA

The authors have addressed my earlier critiques.

Competing Interests: No competing interests were disclosed.

I confirm that I have read this submission and believe that I have an appropriate level of expertise to confirm that it is of an acceptable scientific standard.

\section{Version 1}

Reviewer Report 09 May 2016

\section{https://doi.org/10.5256/f1000research.9007.r13746}

(C) 2016 Hunstad D et al. This is an open access peer review report distributed under the terms of the Creative Commons Attribution License, which permits unrestricted use, distribution, and reproduction in any medium, provided the original work is properly cited.

\section{David A Hunstad}

Division of Pediatric Infectious Diseases, Washington University in St Louis, St Louis, MO, USA Andrew Janowski

Department of Pediatrics, Washington University in St Louis, St Louis, MO, USA

Ganapathi et al describe a case series of 16 patients who received a total of 19 courses of cidofovir for adenovirus infection. The authors provide virological data to demonstrate a response to cidofovir therapy, and provide additional data regarding renal function and co-administration of renal toxic medications. Ganapathi et al. provides unique data regarding the peak creatinine level 
during therapy with cidofovir, though this value is difficult to interpret given the confounding factors of other nephrotoxic agents.

This case series is an incremental addition to the number of published cases of pediatric patients infected by adenoviruses who were treated with cidofovir. The manuscript follows a similar format to the case series reported by Bhadri et al. (2009), with presentation of the effects on renal function, and a summarization of the literature.

The authors should summarize outcomes from all other case series to provide updated overall mortality and nephrotoxicity of CDV-treated adenovirus infections in hematopoietic stem cell transplants, solid organ transplants, or other patients. Such analyses may enhance the timeliness and utility of this manuscript.

In the discussion, the authors describe the potential benefit of cidofovir in solid organ transplant patients, in conjunction with reduced immunosuppression. However, without a comparative cohort of children who did not receive cidofovir or reduced immunosuppression, it would be difficult to draw this conclusion from the data presented. In addition, the authors do not discuss two interesting published papers. Humar et al. (2005) described a cohort of adult SOT patients with adenovirus viremia who did not receive cidofovir; all had spontaneous resolution of viremia with no deaths. In contrast, Seidemann et al. (2004) reported that 3 out of 5 pediatric SOT with adenoviremia (one also had a HSCT) died despite receiving cidofovir. The authors should absolutely cite these studies, especially Seidemann as it included pediatric SOT patients. In addition, the authors should highlight whether the positive outcomes in their SOT population are aligned with the adult study by Humar et al. (and the conclusion in that paper that treatment may not be necessary for adenoviremia in SOT patients); and contrast their results with those of Seidemann et al.

Specific comments

1. Methods: The authors should report their study site's protocol for testing for adenoviruses, specifically, whether testing for adenoviruses was only performed on symptomatic patients, or if high-risk patients were routinely screened for adenovirus.

2. Methods: Probenecid is often co-administered with cidofovir. The authors should report if this drug was used in any of the courses of cidofovir, and what dosages. If probenecid was not used in all courses, the authors should analyze their data to investigate the role of probenecid in reducing nephrotoxicity.

3. Methods: It is not clear if adenovirus testing is completed on whole blood or serum. Later in the results, the authors state whole blood, but this should be explained in the methodology.

4. Methods: The authors should define pre-treatment/peak/post-treatment creatinine levels in their methodology. In addition, what defines pre-treatment creatinine? Is that the creatinine on the day of initiation of cidofovir? One week prior? Baseline from months prior? Defining this value is essential to the author's statistical analysis.

5. Table 1: Patients should be ordered by diagnosis for better organization (as opposed to patient number); for example, the hematopoietic stem cell transplant patients should be grouped together, then the solid organ transplant patients should be grouped together. A 
column of outcomes should be added for greater clarity of which cases resulted in mortality, instead of the usage of an asterisk.

6. Results: Much of the information stated in the paragraph starting "Of the 19 courses prescribed" is redundant with the data presented in table 1, including the number of courses involving viremia, pneumonia, and GI symptoms. This paragraph can be consolidated, and the case characteristics presented as already implemented in Table 1.

7. Results: The authors should include a description of the patient's response to cidofovir in Table 1. This would provide better organization and clarity in interpreting which characteristics were associated with response (or lack thereof) to therapy. It also would make the paragraph "We further examined the 16 blood-positive..." redundant, and this could be consolidated.

8. Figure 1: This figure might be easier to interpret if it were separated, for example, into two graphs of HSCT and SOT/other patients. It is difficult to follow 16 distinct virological lines in the current graph. In addition, the color choices for patients 4, 9, and 13 are too similar.

9. The statistical analysis of increased risk of renal dysfunction when receiving $1 \mathrm{vs}>2$ renal toxic medications should be omitted as currently presented. The authors do not describe whether the additional nephrotoxic agents were co-administered at the same time or for what duration. These are critical confounding variables that are necessary for interpretation of whether nephrotoxicity was associated with increased administration of additional nephrotoxic agents. Given that this review is retrospective, such information may or may not be available.

10. Figure 2 is a somewhat awkward way to describe SCr before and after CDV therapy. In addition, it is unclear how, if only $64 \%$ of patients with elevated SCr returned to baseline, the mean SCr after CDV was equal to baseline (in the right bar of Figure 2). The table describes the $\mathrm{SCr}$ data adequately, and Figure 2 could therefore be omitted.

11. The publication search criteria used to generate Table 3 should be explained in the methods. In their literature review, the authors should incorporate other pediatric adenovirus-cidofovir case reports and series that were not reviewed in this manuscript, including: Seidemann et al. (2004), Leruez-Ville et al. (2004), Nagafuji et al. (2004).

12. At least brief mention of the advent of brincidofovir for treatment of these patients with much reduced nephrotoxicity would be warranted.

\section{References}

1. Bhadri VA, Lee-Horn L, Shaw PJ: Safety and tolerability of cidofovir in high-risk pediatric patients. Transpl Infect Dis. 2009; 11 (4): 373-9 PubMed Abstract | Publisher Full Text

2. Humar A, Kumar D, Mazzulli T, Razonable RR, et al.: A surveillance study of adenovirus infection in adult solid organ transplant recipients.Am J Transplant. 2005; 5 (10): 2555-9 PubMed Abstract | Publisher Full Text

3. Seidemann K, Heim A, Pfister ED, Köditz H, et al.: Monitoring of adenovirus infection in pediatric transplant recipients by quantitative PCR: report of six cases and review of the literature.Am J 
Transplant. 2004; 4 (12): 2102-8 PubMed Abstract | Publisher Full Text 4. Leruez-Ville M, Minard V, Lacaille F, Buzyn A, et al.: Real-time blood plasma polymerase chain reaction for management of disseminated adenovirus infection.Clin Infect Dis. 2004; 38 (1): 45-52 PubMed Abstract | Publisher Full Text

5. Nagafuji K, Aoki K, Henzan H, Kato K, et al.: Cidofovir for treating adenoviral hemorrhagic cystitis in hematopoietic stem cell transplant recipients.Bone Marrow Transplant. 2004; 34 (10): 909-14 PubMed Abstract | Publisher Full Text

Competing Interests: No competing interests were disclosed.

\section{We confirm that we have read this submission and believe that we have an appropriate level of expertise to confirm that it is of an acceptable scientific standard, however we have significant reservations, as outlined above.}

Author Response 05 Dec 2016

Lakshmi Ganapathi, Boston Children's Hospital, USA

Addressing Dr Hunstad's and Dr Janowski's report, in the new version of the manuscript we have clarified the following in the methods section: 1) The protocol for testing for adenovirus infection, 2) Usage of probenecid and dose (it was used in all patients who received cidofovir), 3) how we defined pre-treatment creatinine and 4) the publication search criteria. While we mention the use of concomitant medications that are known to render nephrotoxicity, we agree that the analysis is confounded by several factors and hence have omitted the statistical analysis of increased risk of renal dysfunction when receiving 1 versus $>1$ nephrotoxic medications.

In the discussion section, we have expanded our discussion on the potential benefit of cidofovir in solid organ transplant recipients, and compare the findings of Humar et al (a study in which patients were all adults) with the findings reported by Seidemann et al and Leruez-Ville et al. As such, we added the latter two studies to table 3 for an updated literature review. However, we did opt to leave out the study by Nagafuji et al as recommended by the reviewers. The study by Nagafuji et al focused primarily on adult HSCT recipients and included only one pediatric patient. We feel that given much larger pediatric specific case series of HSCT recipients with adenovirus infection, this one case reported adds little. On the other hand, we did include case reports in the solid-organ patient literature review given the paucity of data in this population. We have also made a brief mention regarding the advent of brincidofovir.

We updated table 1 to include include a column of outcomes. While we recognize that other changes as recommended by the reviewers may contribute to better organization of text and data, we did not make too many other changes to table 1 or figure 1 given that these changes are stylistic and not necessarily content relevant.

Competing Interests: None 
Reviewer Report 04 May 2016

https://doi.org/10.5256/f1000research.9007.r13587

(c) 2016 Bergelson J. This is an open access peer review report distributed under the terms of the Creative Commons Attribution License, which permits unrestricted use, distribution, and reproduction in any medium, provided the original work is properly cited.

\section{Jeffrey Bergelson}

Department of Pediatrics, University of Pennsylvania School of Medicine, Philadelphia, PA, USA

This paper reports the use of cidofovir for treatment of adenovirus infection in 16 pediatric patients, and provides detailed information about the patients' clinical status, blood viral loads, and renal function throughout the treatment course.

I have only one major point for the authors to consider. Most patients survived, and a number had decreases in viral load within several weeks of the start of cidofovir therapy. However, the patient group was quite heterogeneous (one case had cerebral palsy, but no apparent immune dysfunction); in some cases the viral loads rose for a long time before they began to fall; and 4 of the 7 patients who had undergone allogeneic stem cell transplant died. I think the discussion should point out explicitly that it is difficult to conclude from these data whether or not cidofovir provided any clinical benefit.

Minor points:

1. Clarify the assay used for viral loads. Is it the Argene assay, or an in-house assay using some components of the Argene kit?

2. Clarify the definition of "viral response". Is it a 10-fold decrease in titer?

3. Perhaps mention briefly why the asymptomatic patients were treated with cidofovir. It appears that one was a lung transplant patient (considered to be at high risk) and one a SCT patient with recurrent viremia.

4. Figure 2 adds little.

Typos, spelling, glitches:

Page 3. "These associated adverse effects"; THIS adverse EFFECT.

Page 3. "1 $\mathrm{mg} / \mathrm{kg}$ three times" WEEKLY

Page 3. "Respiratory tract"; italicize

Page 3. "The peak SCr during therapy was used to calculate the number..." should be deleted.

Table 1. "multivisCeral"

Page 5. "Respiratory symptoms were the most common presentation in 10 courses"; should be "... most common presentation (10 courses).."

Page 5. "Two courses were prescribed... respectively"; should be One course was prescribed in asymjptomatic $x$, and one in $y$. 
Page 5. "improvement with viral clearance, however"; comma should be a semicolon.

Page 6. ".. one third died all of which"; ? ...died, all of WHOM..."

Competing Interests: No competing interests were disclosed.

\section{I confirm that I have read this submission and believe that I have an appropriate level of expertise to confirm that it is of an acceptable scientific standard.}

Reviewer Response ( ) 05 May 2016

Jeffrey Bergelson, University of Pennsylvania School of Medicine, Philadelphia, USA

Because the paper provides no evidence about efficacy, the abstract should be changed, as well as the discussion. "....was associated with viral response and clinical improvement" should be softened.

Competing Interests: None

\section{Author Response 05 Dec 2016}

\section{Lakshmi Ganapathi, Boston Children's Hospital, USA}

Addressing Dr Bergelson's report, in the new version of the manuscript we have expanded our discussion to point out more explicitly that at least based on our data, while we did observe clearance of viremia in several patients overall clinical benefit was somewhat harder to conclude. We have also mentioned the rationale behind treating asymptomatic patients (both treated patients were considered to be at high risk for complications).

In the methods section, we have clarified the following: 1) The assay used to determine viral load, 2) What we defined as viral response.

We have also corrected the minor spelling and punctuation issues.

We agree with both Dr Bergelson and Dr Hundstad that Figure 2 adds little and have removed it from this version of the manuscript

Competing Interests: none

Reviewer Report 03 May 2016

https://doi.org/10.5256/f1000research.9007.r13586

(C) 2016 O'Ryan M. This is an open access peer review report distributed under the terms of the Creative Commons Attribution License, which permits unrestricted use, distribution, and reproduction in any medium, provided the original work is properly cited. 


\begin{abstract}
Miguel O'Ryan
Institute of Biomedical Sciences, Faculty of Medicine, Universidad de Chile, Santiago, Chile

This is a well written descriptive, retrospective study of a case series of immunocompromised children receiving cidofovir for treatment of mostly probable, few definite or asymptomatic adenovirus infections. Children had different underlying diseases including a few with solid organ transplantation. The series review is transparent, showing viral and clinical evolution as well as renal compromise in association with treatment. Most children cleared adenovirus with treatment but as the authors point out, also in association with improved immunity. Most but not all children clearing the virus improved clinically, a few died despite clearing the virus. Transient nephrotoxicity occurred in near $50 \%$ of children, but this occurred in association with other nephrotoxic treatments and was not a major problem. This review adds to the rather limited number of currently available series and although not providing any definite conclusion (clinicians will still have to balance several variables before deciding to use cidofovir or not) it adds helpful information for treating physicians that have to make these hard decisions.
\end{abstract}

Competing Interests: No competing interests were disclosed.

I confirm that I have read this submission and believe that I have an appropriate level of expertise to confirm that it is of an acceptable scientific standard.

\title{
Comments on this article
}

\section{Version 1}

\section{Reader Comment 07 Dec 2016}

Karoly Toth, Saint Louis University Health Sciences Center, USA

The readers may be interested to note that several compounds were found to be efficacious against disseminated adenovirus infection in the permissive Syrian hamster animal model. Some of these compounds are already approved for other indications, while others are undergoing clinical trials. The references to these publications are provided below.

Toth et al. 2008. Hexadecyloxypropyl-cidofovir, CMX001, prevents adenovirus-induced mortality in a permissive, immunosuppressed animal model. Proc Natl Acad Sci U S A 105:7293-7297.

Diaconu et al. 2010. Human adenovirus replication in immunocompetent Syrian hamsters can be attenuated with chlorpromazine or cidofovir. Journal of Gene Medicine 12:435-445.

Ying et al. 2014. Ganciclovir inhibits human adenovirus replication and pathogenicity in permissive immunosuppressed Syrian hamsters. Antimicrob Agents Chemother 58:7171-7181.

Tollefson et al. 2014. Cidofovir and brincidofovir reduce the pathology caused by systemic infection with human type 5 adenovirus in immunosuppressed Syrian hamsters, while ribavirin is largely ineffective in this model. Antiviral Res 112:38-46.

Toth et al. 2015. Valganciclovir inhibits human adenovirus replication and pathology in permissive immunosuppressed female and male Syrian hamsters. Viruses 7:1409-1428. 
Competing Interests: No competing interests were disclosed.

The benefits of publishing with F1000Research:

- Your article is published within days, with no editorial bias

- You can publish traditional articles, null/negative results, case reports, data notes and more

- The peer review process is transparent and collaborative

- Your article is indexed in PubMed after passing peer review

- Dedicated customer support at every stage

For pre-submission enquiries, contact research@f1000.com 